\title{
ULTRASONIC WAVE PROPAGATION IN CYLINDRICAL VESSELS AND IMPLICATIONS FOR ULTRASONIC REACTOR DESIGN
}

\author{
T. Mutasa ${ }^{\mathrm{a}}$, A. Gachagan ${ }^{\mathrm{a}}$, A. Nordon ${ }^{\mathrm{b}}$, R.L. O’Leary ${ }^{\mathrm{a}}$ \\ ${ }^{a}$ Centre for Ultrasonic Engineering University of Strathclyde, G1 1XW, Glasgow, UK. \\ ${ }^{b}$ Centre for Process Analytics and Control Technologies, University of Strathclyde, Glasgow, G1 $1 X W$
}

\begin{abstract}
Reactors in which processes are enhanced by ultrasound are hampered by the lack of a theoretical framework on their design. Simulation results of ultrasonic wave propagation in a cylindrical geometry are presented in this work, which are then used to develop guidelines for the design of ultrasonic reactors. These guidelines are used to design a new type of reactor with a novel geometry, operating at a frequency of $27 \mathrm{kHz}, 39 \mathrm{kHz}$ and $82 \mathrm{kHz}$. This reactor is characterized using Weissler's reaction dosimetry.
\end{abstract}

Index Terms-Reactor Design, Resonance, Cavitation, Ultrasound

\section{INTRODUCTION}

Ultrasound applied to liquids at sufficiently high displacement levels, can cause the formation of cavities within the liquid which grow, become unstable and collapse leading to a concentrated release of energy in a process called inertial cavitation. This energy release has been shown to provide benefits for many processes either chemically or mechanically.[1] As such, a lot of interest has been shown in developing these observations for commercial application in industrial processing. For further development of this technology, a theory needs to be developed on the design of sonochemical reactors.

Sonochemical processing is a multidisciplinary study composed of chemical dynamics which govern the processing, bubble dynamics which determine occurrence of cavitation and strength of cavitation, as well as acoustic dynamics which define the distribution of acoustic pressure within the vessel.[2] Studies conducted to investigate the behavior of various configurations of sonication reactors have mainly relied upon empirical evidence, as such significantly lack repeatability and thereby fail to provide strong causal relations. Recently it has been observed that multiple frequency insonication does not have a straight forward relationship with increased efficiency, with some conditions resulting in improved efficiency while others show decreased efficiency [3]. In a similar manner both operational frequency and height of the reactor have been shown to improve efficiency in some conditions, while other publications will show them to decrease the efficiency of the system.[4]-[6]

Neppiras et al showed that inertial cavitation occurs in a region when the local pressure exceeds a certain threshold, governed by several system parameters including the excitation signal and the liquid properties. Other investigators have shown that as the pressure level within a vessel increases, so does the observed ultrasonic effect [5]. From this relationship, it is a considered a reasonable approach to simulate the pressure within a vessel and assume a direct correlation between cavitation intensity and local pressure. Such a relationship has been supported through field mapping using sonoluminescence with the brightest areas being regions of highest predicted pressure. It has also been observed by means of aluminium foil tests with the areas suffering most degradation being cavitation regions [7]-[8]. At cavitation pressure levels the wave propagation is considered nonlinear and hence the standard linear wave propagation equation may not be strictly applicable. Moreover, the commencement of cavitation leads to the proliferation of bubbles which contribute to increased scattering. This has been simulated using a simple attenuation model, and the pressure field observed has not been very different from that predicted by the linear wave equation [9].

It is the intention of this paper to present work focused on the acoustic dynamics of sonochemical systems developed through numerical modeling using finite element methods. Insights from modeling are then used to design a new type of ultrasonic reactor.

\section{SIMULATION OF REACTOR}

\section{A. Simulation Procedure.}

In order to understand acoustic pressure profiles for a given vessel geometry, finite element simulations were performed using PZFlex [10]. The Model was configured to predict the propagation of waves in a reactor vessel and the type of wave field distribution developed. The reactor vessel considered was prismatic with its geometry modeled in two dimensions by the axial cross section with pressure assigned in the radial direction. Pressure was applied as a forcing function at a region whose size was determined by the size of the radiator. Preliminary simulations showed that different shaped and 
sized vessels each exhibited different field patterns, hence it was decided to focus on one shape, the simplest geometry (circular) and then generalize the theories developed for that geometry onto more complex geometries. Figure 1 details a schematic of the model, it comprises a cylindrical solid wall containing a liquid medium and bounded externally by air. From Figure 1 the diameter of the liquid component of the model is $D$ with the thickness of the wall material being $3 \mathrm{~mm}$, in the first instance only a single source of excitation will be considered.

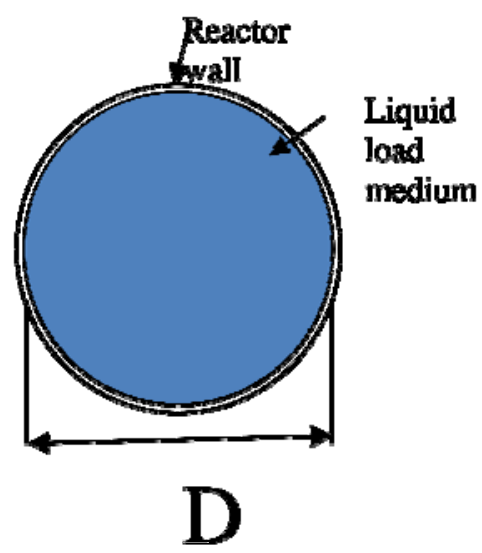

Figure 1 Schematic diagram of the model configuration

In order to determine the natural propagation characteristic of this geometry, a forcing function was applied to a small section of the interface between the reactor wall and the liquid medium. This function had to have sufficient bandwidth to cover the frequencies of interest and hence a chirp signal with frequency content from $10 \mathrm{kHz}$ to $150 \mathrm{kHz}$ was used. Element size for the model was such that for a frequency of $200 \mathrm{kHz}$, each element was at least $\lambda / 20$. The forcing function applied for $10 \mu \mathrm{s}$ and the response allowed to decay naturally. The response to the forcing function was then be measured at all possible points as determined by the model resolution. Assuming the propagation characteristics form a linear time invariant channel, the propagation characteristic of that geometry at that scale can be found by computing the frequency response function at all points.

$$
\begin{gathered}
\boldsymbol{H}(f, r)=\frac{Y(f, r)}{X\left(f, r_{0}\right)} \\
\boldsymbol{Y}(f, r)=\mathcal{F}[y(t, r)] \\
\boldsymbol{X}\left(f, r_{0}\right)=\mathcal{F}\left[x\left(t, r_{0}\right)\right]
\end{gathered}
$$

$x\left(t, r_{0}\right)$ is the excitation signal applied at position $r_{0}$ while $y(t, r)$ is the response to that excitation at point $r$. The results show a large variation in response at all points in the volume with some regions showing a high response and others showing very little power transfer. Figure 2 compares the response of the vessel at $20 \mathrm{kHz}$ and $40 \mathrm{kHz}$ for a vessel diameter or $120 \mathrm{~mm}$ with a $3 \mathrm{~mm}$ wall of stainless steel. Figure 2 illustrates that variation is influenced by frequency with different locations resonating at different frequencies, but there does appear to be a central tendency over the numerous responses which can be estimated by using the median pressure over all points at a particular frequency. This leads to the derivation of the median pressure frequency response, which was used to determine the performance of the system $P_{M}$, which will be employed as a metric when evaluating reactor vessel designs presented in the subsequent section of this paper.

$$
P_{M}(f)
$$

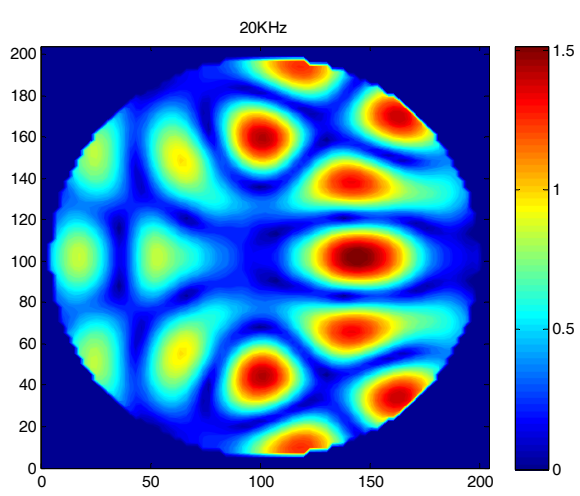

(a)

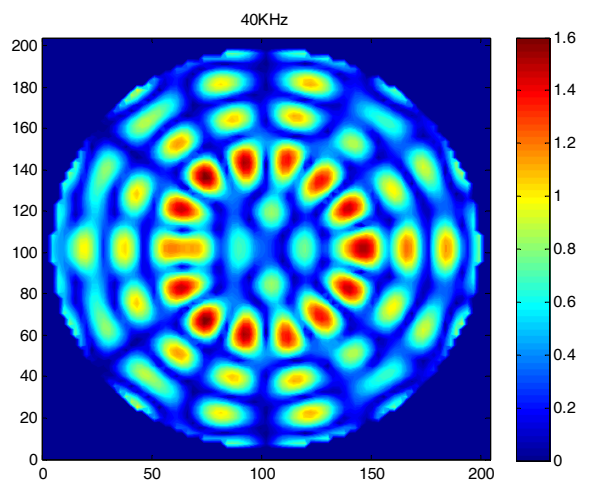

(b)

Figure 2 Comaprison of vessel frequency response for $120 \mathrm{~mm}$ diameter stainless steel vessel at (a) $20 \mathrm{kHz}$ and (b) $40 \mathrm{kHz}$

\section{B. Reactor Scale, Frequency and Chemical Composition Effects.}

The median pressure frequency response shown in Figure 3 demonstrates a distinct resonant profile with regularly spaced peaks. These resonances are indicative of specific modes of vibration within the fluid as they coincide with a regularly distributed pressure profile resembling geometric shapes. The presence of these resonant modes shows that there are particular frequencies for which a reactor design is optimized, resulting in a more efficient mode of operation. It is of interest for the efficient transfer of sonochemical technology to industrial processes, that larger scale designs be considered. In this regard the size of vessels was varied and the median pressure frequency response function calculated. The calculated function showed the same general trend, but with the resonances occurring at different frequencies.

It is important to note that the nature of any given reactor will be dynamic, i.e. during the ensuing reaction the acoustic properties of the reactor will be dynamic as the reaction precursors are consumed by the reaction. In order to investigate this effect. The simulation procedure was repeated and the phase velocity of the liquid medium within the reactor was varied in the range $1000 \mathrm{~ms} /$ to $1600 \mathrm{~m} / \mathrm{s}$ The calculated 
median pressure profile for different phase velocities showed a similar response to that calculated for different diameters but with a reversed effect where an increase in phase velocity was comparable to a decrease in diameter or frequency. The frequency transfer response at any point can be used to derive a specific impulse response of that particular channel.

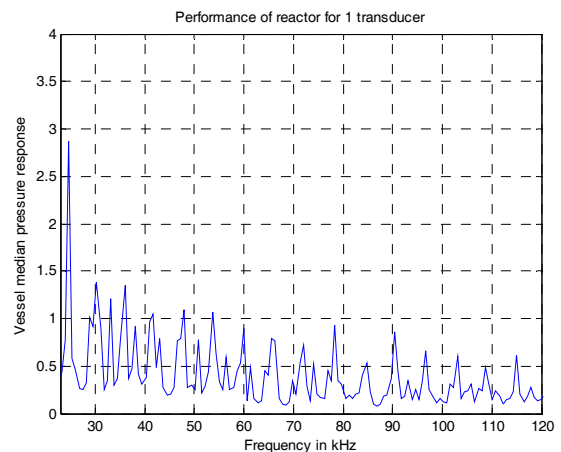

Figure 3 Median Pressure frequency response function, showing distinct peaks at resonance.

Changing the size or material properties of the fluid inside the reactor will change the impulse response of the channel by either expanding or contracting the temporal axis, hence there exists a single deterministic function that relates the median pressure profile to frequency, phase velocity and dimensions for shapes with circular cross-section. Such a function can then be optimized, subject to the appropriate constraints, aiding the design of reactor vessels.

$$
P_{M}(f, c, D)=P_{M}(\gamma)
$$

Where

$$
\gamma=f \cdot \frac{D}{c}
$$

Under such conditions, optimization often leads to a design resonating on the fundamental mode of vibration of the system.

\section{Multiple sources of ultrasound.}

The intuitive approach to the design of larger scale sonochemical reactors is the addition of more sources of ultrasound to the vessel. The results of the simulations presented in the preceding section for single sources excitation exhibited complex self interaction effects. It was therefore it was desirable to investigate the effect on reactor performance of adding multiple sources. Calculations were made through the superposition of the same response to itself after some spatial reorientation.

$$
P_{T}(f, r, \theta)=\sum_{j=1}^{n} P\left(f, r, \alpha_{j}\right)
$$

Where $P_{T}$ is the overall pressure from multiple sources each having equal pressure frequency response $P$ at location determined by circular co-ordinates $r$ and $\theta . \alpha_{j}$ is given by Equation 8 - where $\theta_{j}$ is the angular position of the transducer outside the reactor walls.

$$
\alpha_{j}=\theta+\theta_{j}
$$

From the calculated pressure distribution profile new median pressure frequency response functions where computed for different reactor configurations. The results show that multiple sources results in mode selective behavior with the selected modes determined by the phasing strategy used. The most important observation however was that addition of more sources did not result in a linear increase in median pressure response. It was only at resonance that a linear relationship exists with the number of transducers probably due to the geometric mode shapes. At certain frequencies the addition of more sources resulted in a cancellation effect and a decline in median pressure. Figure 4 shows the overall effect of adding more sources and a distinct diminishing returns relationship.

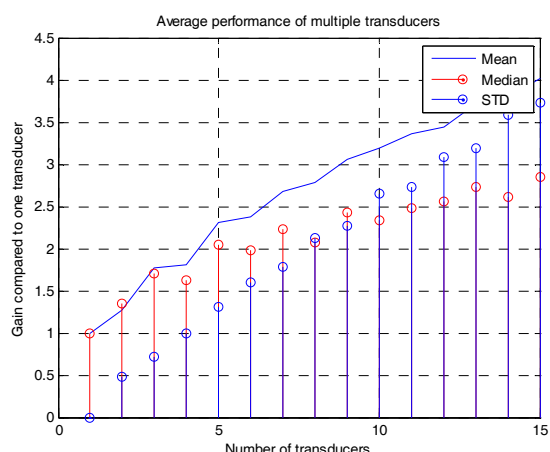

Figure 4 Overall median pressure gain for multiple transducers referenced to the response for a single transducer.

\section{Reactor Design.}

Results from optimization algorithms indicate that smaller vessels, and their lowest resonant modes, are optimal designs. It is however the objective of the work to have a larger vessel to accommodate the requirement for industrial scale processing. Alternate geometries were investigated using the methodology described. The final design, the implementation of which will be described in the following section, is an annulus. The advantage of this design is that the resonant dimension does not have a strong effect on cross-sectional area and therefore a large area could be processed while remaining in the first resonant mode. In addition, this geometry is characterized by very sharp resonances. Optimization was achieved by maximizing the following objective function. Where $\gamma$ is a scaling factor for the importance of size.

$O=D^{2 \gamma}\left(P_{M}\left(f_{1}, c, D\right)+P_{M}\left(f_{2}, c, D\right)+P_{M}\left(f_{2}, c, D\right)\right)$

The reactor design was constrained such that commercially available transducers would be employed, limiting the design to $27 \mathrm{kHz}, 39 \mathrm{kHz}$ and $82 \mathrm{kHz}$. In addition, the reactor would be specified for liquid media exhibiting a phase velocity from 1100 to $1500 \mathrm{~m} / \mathrm{s}$. this resulted in the final reactor design of the following dimensions: $350 \mathrm{~mm}$ for outer wall diameter and $200 \mathrm{~mm}$ for inner wall diameter. Wall material choices where made based on a desire for resistance to chemical corrosion and good ultrasound transfer characteristics. Stainless steel was chosen based on its resistance to chemical corrosion. To limit the energy loss a thin wall was desired, but it had to be thick enough to withstand machining of flat surfaces for transducer attachment. $5 \mathrm{~mm}$ thickness was the value chosen. The inner wall was also chosen to be stainless steel, but it was thinner to enhance temperature control through this interface. Figure 5 details a photograph of the implemented design. 


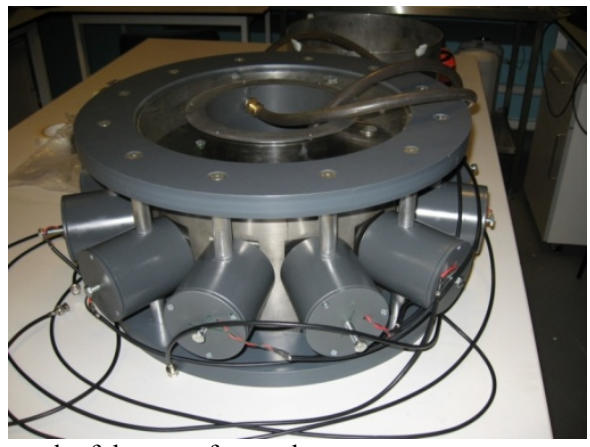

Figure 5 Photograph of the manufactured reactor

\section{E. EXPERIMENTAL EVALUATION OF REACTOR}

Sonication energy is achieved by means of a proprietary power supply comprising an H-bridge switching circuit for the high power, a filter and a transformer for impedance matching to the transducers. Xilinx Spartan 3E 500K FPGA controls the switching signals allowing user defined excitation within the frequency range from $17 \mathrm{kHZ}$ to $120 \mathrm{kHz}$ and an arbitrary phase delay, with a resolution of 1 thousandth of a period. The FPGA is interfaced to a personal computer through a USB interface (Digilent, Pullman, WA). A laboratory mercury thermometer is used to measure the temperature before commencement of sonication, which is found to be $19.7^{\circ} \mathrm{C}$. The performance of the reactor was assessed using Wiessler's reaction. UV spectroscopy employed to determine the concentration of triiodide ions using the Beer Lambert law and a triiodide molar absorbance of $26303 \mathrm{dm}^{3} \mathrm{~mol}^{-1} \mathrm{~cm}^{-1}$ at $355 \mathrm{~nm}$ [11].

The experimental procedure was conducted by insonicating a $0.1 \mathrm{M}$ solution of Potassium Iodide (KI) solution using all 12 transducers. $10 \mathrm{ml}$ aliquots of KI were removed from the reactor at 5 minute intervals for a total time of 60 minutes and the whole experiment was repeated twice. Ultrasonic energy was computed by monitoring the temperature of the KI solution over time and knowledge the specific heat capacity and the mass of water used in the experiment. Sonochemical efficiency of the reactor was then calculated using the Equation 10 [4]

$$
S_{E}=C_{I} \cdot \frac{V}{E_{U S}}
$$

Where $C_{I}$ is the concentration of triiodide, $V$ is the volume of the whole solution and $E_{U S}$ is the acoustic energy input as calculated from the temperature rise. The sonication of the reactor was found to be $6.5 \times 10^{-10} \mathrm{~mol} / \mathrm{J}$, this is plotted in Figure 6 where the performance of the reactor is compared to results obtained for 4 different reactors described in the literature: A [4], B [12], C [13] and D [14]

The results show that the presented reactor is not as efficient as the other reactors. This might be because it is operating outside resonance.

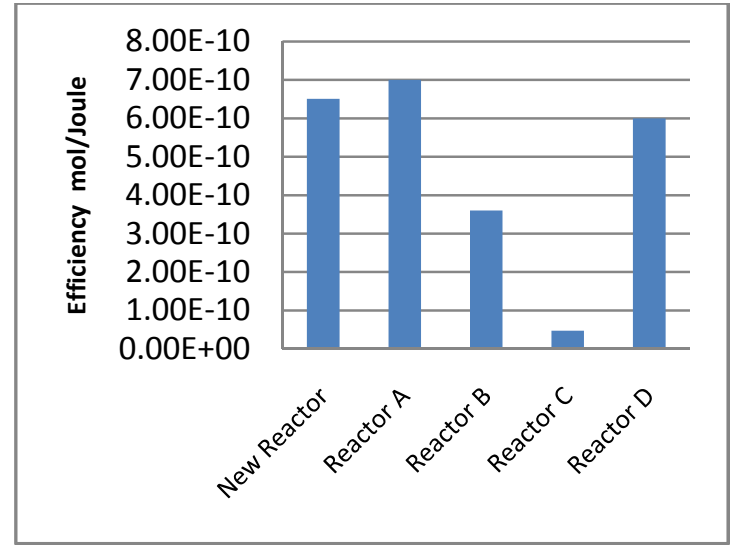

Figure 6 Comparison of reactor sonication efficiency to data available in the literature, A [4], B [12], C [13] and D [14]

\section{CONCLUSIONS}

A study of the acoustic dynamics of reactors is critical for the design of efficiency high power ultrasonic reactor vessels. Finite element simulations show that there is a significant advantage to be gained by operating at resonance. Also, care must be taken when adding multiple transducers to the reactor system since a serious degradation in performance can be observed. Given the high Q-factor of the resonances and the poor performance at frequencies which are not resonant it is paramount that reactor designs operate at resonance. Power transducers are usually narrow band with sharp resonances to achieve large displacement amplitudes and as such also have poor performance outside their resonant zone. The ideal performance will be achieved when the reactor and transducer resonance coincide, however this is difficult to achieve due to tight manufacturing tolerances and instability at the reactor resonances caused by temperature variation in the reactor liquid medium. In light of these findings it expected that a performance compromise may be required, for example sacrificing displacement amplitude to achieve a wider-band but lower power devices, whose operating frequency can be tuned to match that of the reactor. Alternatively, temperature control can be used to modify the phase velocity of the medium and hence the resonant behavior of the reactor so that it coincides with that of the reactor. However this will be a more challenging solution.

\section{REFERENCES}

[1] K.S. Suslick, J. Am Chem Soc, 108, pp. 5641-5642, 1986

[2] L.A. Crum, Ultrasonics Sonochemistry, Vol 2, No 2, 1994

[3] Y. Son, Japanese Journal of Applied Physics, 49, 2010

[4] Y. Asakura, Ultrasonics Sonochemistry, Vol. 15, pp. 244-250, 2008

[5] P.R. Gogate, Ultrasonics Sonochemistry, Vol 10, pp. 325-330, 2003

[6] M.A. Beckett, Journal of Physical Chemistry.,105, pp.3796-3802, 2000

[7] K. Yasui, Ultrasonics Sonochemistry, Vol. 14, pp. 605-614, 2007

[8] K-V Jenderka, Ultrasonics, Vol 44, pp. 401-406, 2006

[9] S. Dahnke, Ind Eng Chem. Res, Vol 37, pp. 848-864, 1998

[10] PZflex, Weidlinger Associates, Inc, Mountain View, CA.

[11] S. Koda, Ultrasonics, Sonochemistry, Vol 10, pp. 149-156, 2003

[12] S. de La Rochebrochard d'Auzay et al, Ultrasonics Sonochemistry, Vol 17 , pp. 547-554, 2010

[13] K.R. Morison, Ultrasonics Sonochemistry, Vol 16, pp.179-183, 2009

[14] S. Merouani, Journal of Hazardous Materials, Vol 178, pp.1007-1014, 2010 